\title{
Abnormal Spontaneous Brain Activity and Functional Connectivity of the Default Mode Network in Patients with Bipolar Disorder
}

\section{Lei Zhao}

Beijing Anding Hospital

Qijing Bo

Beijing Hospital

\section{Zhifang Zhang}

Beijing Hospital

\section{Feng Li}

Beijing Anding Hospital

\section{Yuan Zhou}

Chinese Academy of Sciences

Chuanyue Wang ( $\nabla$ wcyadyy@163.com )

Beijing Anding Hospital https://orcid.org/0000-0002-0781-6710

\section{Research Article}

Keywords: Default mode network, precuneus, bipolar disorder, resting-state functional magnetic resonance imaging, functional connectivity

Posted Date: February 15th, 2021

DOI: https://doi.org/10.21203/rs.3.rs-194210/v1

License: (c) (i) This work is licensed under a Creative Commons Attribution 4.0 International License. Read Full License 


\section{Abstract}

Background: No consistent evidence on the specific brain regions is available in the default mode network (DMN), which show abnormal spontaneous activity in bipolar disorder (BD). We aim to identify this region that is particularly impaired in patients with BD by using several different indices measuring spontaneous brain activity and then investigate its functional connectivity (FC).

Methods: A total of 56 patients with BD and 71 healthy controls $(\mathrm{HC})$ underwent resting-state functional magnetic resonance imaging. Three commonly used functional indices were used to identify the brain region showing abnormal spontaneous brain activity in BD. Then, this region served as the seed region for resting-state FC analysis to identify its functional networks altered in BD.

Results: The BD group exhibited decreased fALFF, ReHo, and DC values in the left precuneus. The BD group had decreased rsFC within the DMN, indicated by decreased resting-state FC within the left precuneus and between the left precuneus and the medial prefrontal cortex. The BD group had decreased negative connectivity between the left precuneus and the left putamen, extending to the left insula.

Conclusions: The findings provide convergent evidence for the abnormalities in the DMN of BD, particularly located in the left precuneus. Decreased FC within the DMN and the disruptive anticorrelation between the DMN and the salience network are found in BD. These findings suggest that the $\mathrm{DMN}$ is a key aspect for understanding the neural basis of BD, and the altered functional patterns of DMN may be a potential candidate biomarker of BD.

\section{Background}

Bipolar disorder (BD) is a progressive neurodevelopmental disorder characterized by emotional disturbance; it affects more than $1 \%$ of the world's population ${ }^{1}$ and $0.6 \%$ of the Chinese population ${ }^{2}$. However, the pathophysiology of BD is poorly understood. Recent advances in the field of neuroimaging have improved our understanding on the neurophysiology of this disease ${ }^{3}$. In particular, the use of resting-state functional magnetic resonance imaging (rsfMRI), abnormal local spontaneous brain activity, and resting-state functional connectivity (rsFC) have been repeatedly observed in patients with $\mathrm{BD}^{4,5}$.

These studies indicate that a brain network, called the DMN, is particularly interested in the studies of BD. The default mode network (DMN) consists of medial prefrontal cortex (MPFC), the posterior cingulate cortex (PCC), the precuneus ${ }^{6}$, the inferior temporal and the superior frontal cortices, the hippocampus, and the parahippocampal cortices ${ }^{7,8}$. When a person is awake, but not actively involved in a goal-directed task, this network is active with a high degree of rsFC between regions ${ }^{9}$. Generally, DMN is involved in multiple cognitive and effective functions, such as emotional processing, mind wandering, recollection of experiences, self-referential mental activity, social behavior, and episodic memory processes ${ }^{6}$, all of which have varying degrees of damage in $\mathrm{BD}^{10}$. Samantha and colleagues ${ }^{11}$ also reviewed the putative mechanisms for DMN-related dysfunction in mental disorders including the BD from two aspects. On one hand, the altered rsFC within DMN indicates that the integrity of DMN is impaired, which may be related to the deficit of working memory and attention, which is often observed in BD. On the other hand, the antagonistic relationship between the DMN and the task positive networks is abnormal. It may be related to the irregular transformation of introspective and extrospective thinking. The disbalance between DMN and salience network (SN) (one of the task positive networks) may be related to emotion, cognitive, and psychomotor symptoms in $\mathrm{BD}^{12}$.

Empirically, the research has consistently and repeatedly reported that different types or stages of BD patients have rsFC abnormalities of DMN ${ }^{13-18}$. For example, one study recruiting drug naive or unmedicated patients with BD-II 
depression showed decreased rsFC of the PCC with the MPFC and the precuneus ${ }^{16}$. Another study found that patients with unmedicated BD during depressive episode showed weaker rsFC in the left MPFC and right precuneus compared with healthy controls ${ }^{19}$. In addition, decreased rsFC between the DMN and the SN was reported in patients with BD, but the sample was composed of patients at different stages of BD (including manic, depressive, mixed, and euthymic phases $)^{20}$. In these previous studies, the researchers often applied the method of independent component analysis (ICA) to identify the DMN as a whole and then analyze the rsFC of the $\mathrm{DMN}^{14,17-19}$, or select specific brain regions as seeds for the rsFC of the DMN on the basis of prior hypothesis ${ }^{12,16,20,21}$. Although promising findings have been obtained in these previous studies, no consistent evidence is found on the specific brain regions in the DMN, thereby showing abnormal spontaneous activity in patients with BD.

In addition to the two methods, other analytical approaches can be applied to the studies of DMN, such as fractional amplitude of low-frequency fluctuation (fALFF) $)^{22}$, regional homogeneity $(\mathrm{ReHo})^{23}$, and degree centrality (DC) ${ }^{24}$. The fALFF reflects the contribution of low-frequency signal strength in the entire frequency range; the ReHo reflects the synchronization of local neural activity, and the DC reflects the importance of a certain voxel in the whole-brain functional connectivity matrix. With a whole-brain search strategy, these indices can identify the abnormality in the brain. Using these indices, the researcher have already found decreased brain activity of DMN in patients with $\mathrm{BD}^{25-27}$. Therefore, the abnormal spontaneous brain activity in the DMN can be unbiasedly identified using these functional indicators. Finding the abnormal rsFC of the DMN in an unbiased approach is possible by using the regions identified by these functional indicators as the seed region in patients with BD. In a previous study, the researchers have found that the MPFC, an important node of the DMN, showed abnormal brain activity as indicated by the ReHo and the DC in patients with bipolar mania ${ }^{28}$.

We attempted to use a whole-brain search strategy to identify the regions particularly impaired in the DMN and to identify the rsFC of the DMN in the patients with BD. Specifically, from the point of view of local spontaneous brain activity and functional network, we used multiple brain functional indices including fALFF, ReHo, and DC to unravel abnormal brain spontaneous activities in BD. Subsequently, using the cluster that showed significant between-group differences as a seed, rsFC analyses, which reflect functional interactions of spatially distributed brain regions, were performed to further characterize the abnormal functional connectivity of the seed region in patients with BD.

\section{Materials And Methods}

\subsection{Participants}

A total of 150 participants were recruited from the community $(85 \mathrm{HCs})$ and the Beijing Anding Hospital (65 patients with $\mathrm{BD}$ ); the sample is the same as in our previous publication ${ }^{29}$. In summary, the patients and HC were matched for age, gender, and education. Twenty-three subjects (nine patients with BD and 14 HCs) were excluded from the final analysis due to excessive head motion (details are shown in the "fMRI data preprocessing"). All patients satisfied the diagnostic criteria of the Structured Clinical Interview for DSM-IV (SCID) ${ }^{30}$. Our inclusion and exclusion criteria were described in our previous study ${ }^{29}$. In this experiment, the Young Mania Rating Scale (YMRS) ${ }^{31}$ scores of all patients were less than 7, thereby indicating that all patients were not in a manic mood state. We focused on patients with BD in a depressive mood state or a euthymic mood state because BD patients spend more than $80 \%$ of their time in a nonmanic mood state. The Hamilton Depression Rating Scale (HAMD) ${ }^{32}$ was used to assess patients' depressive symptoms.

The current study has been approved by the Institutional Review Board of Brain Image Center, Beijing Normal University and Beijing Anding Hospital, Capital Medical University. Written informed consents were obtained from all subjects prior 


\subsection{Fmri Data Acquisition}

All imaging data were acquired at the Brain Imaging Center of the Beijing Normal University by using a Siemens TIM Trio 3T scanner (Siemens, Erlangen, Germany). Resting state functional images (240 volumes) were acquired initially, followed by the T1 images. During scanning, subjects were asked to try not to swallow or move their body, especially their heads. During resting state, subjects were also instructed to close their eyes, relax, and remain awake. Resting state functional images were collected using echo-planar imaging (EPI) sequence, as follows: axial scanning, repetition time $(\mathrm{TR})=2000 \mathrm{~ms}$, echo time $(\mathrm{TE})=30 \mathrm{~ms}$, flip angle $=90^{\circ}$, field of view $(\mathrm{FOV})=200 \times 200 \mathrm{~mm}^{2}$, matrix size $=64 \times 64$, slices $=33$, interlaced scanning, slice thickness $=3.5 \mathrm{~mm}$, and gap $=0.7 \mathrm{~mm}$. T1 images were collected using T1weighted sagittal 3D magnetization-prepared rapid gradient echo (MPRAGE) sequence, as follows: $\mathrm{TR}=2530 \mathrm{~ms}$, TE = $3.39 \mathrm{~ms}$, flip angle $=7^{\circ}, \mathrm{FOV}=256 \times 256 \mathrm{~mm}^{2}$, matrix size $=192 \times 256$, slices $=128$, thickness $=1.33 \mathrm{~mm}$, and voxel size $=1.33 \times 1 \times 1 \mathrm{~mm}^{3}$.

\subsection{Fmri Data Preprocessing}

Resting state fMRI date was preprocessed in the toolbox for the Data Processing and Analysis for (Resting State) Brain Imaging (DPABI v3.1) ${ }^{33}$. The preprocessing steps included removing the first five time points, slice timing, realignment, detrending, removing the head motion effect (using Friston 24-parameter model) ${ }^{34}$, regressing out nuisance covariates (the white matter signals, the cerebral spinal fluid signals, and the global mean signals), normalizing the MNI space (using T1 images), resampling (voxel size $=2 \times 2 \times 2 \mathrm{~mm}^{3}$ ), smoothing (FWHM = $4 \mathrm{~mm}$, only for the fALFF and rsFC analysis), and band-pass filtering $(0.01-0.1 \mathrm{~Hz}$, only for the ReHo, DC, and rsFC analysis). In addition, we used volumebased framewise displacement (FD) ${ }^{35}$ to quantify head motion. FD reflects head motion from one volume to the next, and a volume with FD $<0.2$ indicates a good time point. Mean FD is calculated by the average of the sum of the absolute values of the differentiated realignment estimates (by backwards differences) at every timepoint ${ }^{36}$. Subjects would be excluded if their mean FD exceeded three standard deviations beyond the mean value of the entire sample or the number of good time points was less than 120.

\subsection{Fmri Data Analysis}

First, three indices of the fALFF, the ReHo, and the DC were used to unravel abnormal brain activities in the patients with BD. Subsequently, using the overlapped cluster of the significant brain region found in the analysis as seed, rsFC analysis was performed to explore possible brain regions, which showed abnormal rsFC with the overlapped cluster in patients with BD. Finally, two additional strategies were used to reidentify the seed region and then confirmed our rsFC analysis.

fALFF, ReHo, and DC calculation. Using the preprocessed resting state fMRI data, we calculated the fALFF, ReHo, and DC values for each subject in DPABI v3.1. All values were calculated at voxel level. The fALFF value was calculated using the signal strength of the low frequency range (i.e., $0.01-0.1 \mathrm{~Hz}$ ) to divide the detectable entire frequency range 22. The ReHo value, also called the Kendall's coefficient of concordance (KCC), of the time series of a given voxel with its nearest neighbors (27 voxels were considered) was calculated to generate individual ReHo maps ${ }^{23}$. The DC value of a certain voxel was the sum of the Pearson's correlation coefficients of all possible pairs of voxels (correlation threshold 
of $r_{0}$ was set at 0.2$)^{24,37}$. Lastly, the fALFF, ReHo, and DC maps for each subject were converted into Z-score maps, respectively. For the ReHo and DC maps, smoothing (FWHM $=4 \mathrm{~mm}$ ) was performed.

Functional connectivity analysis. This analysis was performed in DPABI v3.1. The overlapped brain region, which showed significant group differences in fALFF, ReHo, and DC values between patients with BD and HC, was used as a seed in the following functional connectivity analysis. First, time series of each voxel was extracted within the seed. Second, the mean time series of the seed was calculated by averaging each voxel's time series. Third, the Pearson correlation coefficients of the mean time series of the seed and all other voxels' time series were calculated. They were used to construct each subject's rsFC map. Finally, each subject's rsFC map was converted into Z-score map.

\subsection{Statistical Analysis}

Two sample $T$ test or chi-square tests were used to compared the differences between the patients with BD and the HC in demographic (including age, gender, education, and mean FD) and clinical (i.e. HAMD scores) variables in SPSS 20.0.

For each functional index (i.e., the fALFF, the ReHo, the DC, and the rsFC), analyses of the differences between the two groups were conducted in SPM12 (Wellcome Department of Cognitive Neurology, London, UK). In these analyses, twosample $T$ tests were used with age, gender, education, and mean FD controlled. The significance level was set at the combination of voxel-level uncorrected $P<0.001$ and cluster-level Family Wise Error (FWE)-corrected $P<0.05$. These statistical analyses were limited in a pre-made gray mask. The gray mask was calculated by the average of the gray mask segmented from each subjects' T1 images.

\subsection{Confirmation Analyses Of The Rsfc}

To validate the findings obtained in the rsFC analysis, we used two strategies to confirm the findings. (i) We used the significant cluster obtained in the group analyses of the fALFF and the DC, but not the ReHo (due to the negative finding in a strict statistical threshold) to calculate the overlapped cluster. The overlapped cluster was then used as a seed in the rsFC analysis. (ii). We attempted to map our interested brain regions identified in the analysis of the fALFF, ReHo, and $\mathrm{DC}$ to the Brainnetome atlas ${ }^{38}$. Then, we selected the corresponding brain region of the atlas as seeds for further rsFC analysis. This method reduces the subjective bias in selecting the seed region and makes our findings more likely to be replicated by future studies.

\section{Results}

\subsection{Demographic and Clinical characteristics}

Table 1 summarizes the demographic and the clinical data of all subjects. The two groups were comparable in terms of age, gender, education, and mean FD (all $P$-values $>0.05$ ). The HAMD scores of the BD group were significantly higher than those of the $\mathrm{HC}$ group $(T=7.69, P<0.001)$. As for drug use, the majority of patients was treated with at least one type of psychotropic medication (Table 1). 
Table 1

Demographic and clinical feature of participants

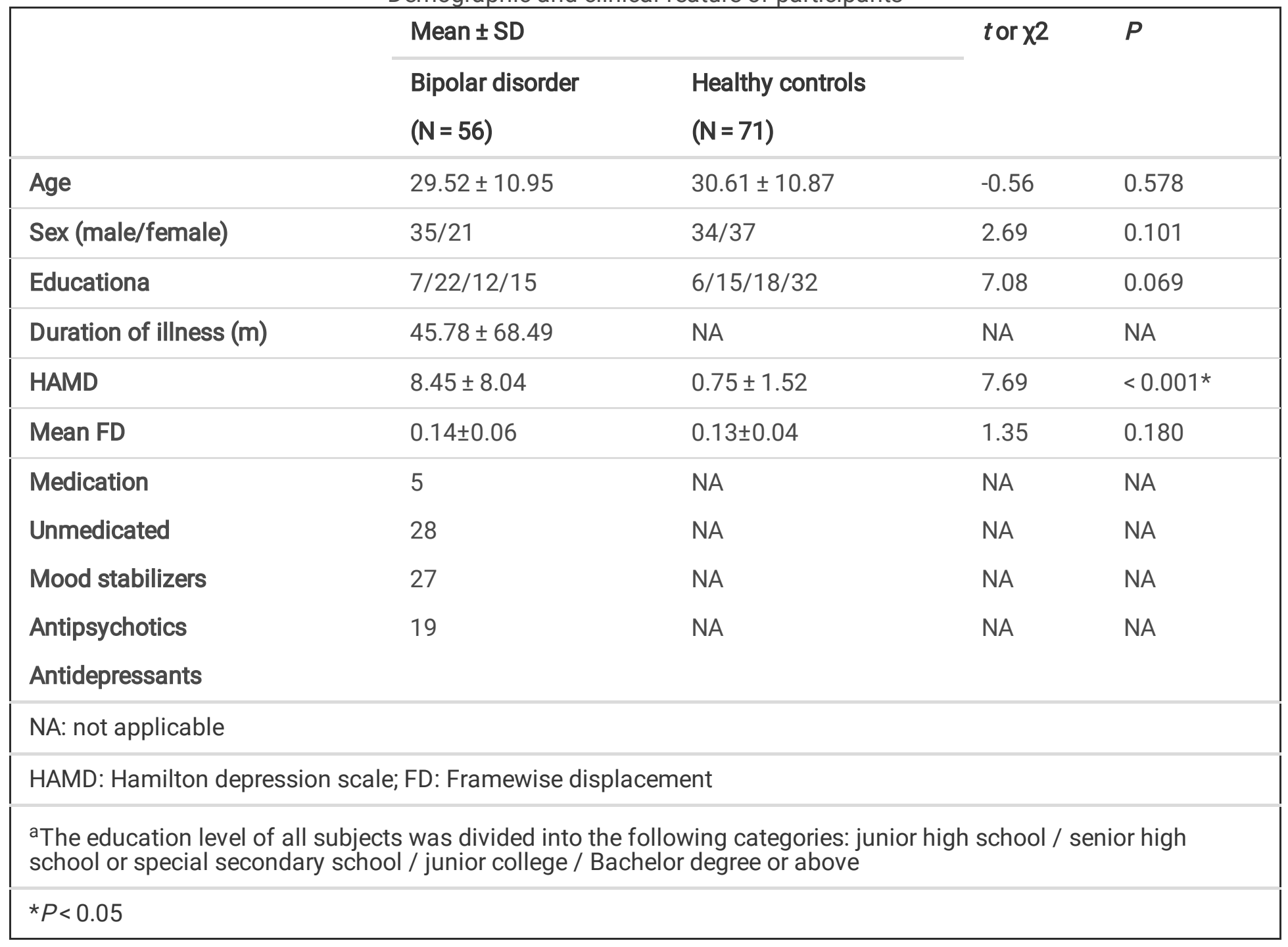

\subsection{Falff, Reho And Dc Analyses}

The fALFF, ReHo, and DC map within each group are shown in Fig. 1-A. The whole-brain analysis found that the BD group showed significantly lower fALFF and DC in the left precuneus. As for the ReHo, though the peak voxel reached the significant level of voxel-level FWE-corrected $P<0.05$, the largest cluster, where the peak voxel was located did not reach our significant level. Thus, we used a relatively less stringent criteria for our ReHo data analysis, i.e., we regarded the largest cluster as an interesting cluster ( $p<0.001$, cluster-level uncorrected). Then, the significant clusters were overlapped, and the common overlapped cluster consisted of 47 voxels, located in the left precuneus (Fig. 1-B and Table 2). 
Table 2

Between-group differences in the fALFF, ReHo and DC analyses between the patients with BD and the healthy controls

\begin{tabular}{|lllllllll|}
\hline & & $\begin{array}{l}\text { Brain } \\
\text { region }\end{array}$ & Hemisphere & BA & $\begin{array}{l}\text { MNI } \\
\text { coordinates }\end{array}$ & $\begin{array}{l}\text { Peak T } \\
\text { values }\end{array}$ & $\begin{array}{l}\text { Cluster } \\
\text { size }\end{array}$ & $\begin{array}{l}\text { Cluster-level } \\
P_{\text {FWE }}\end{array}$ \\
\hline fALFF & $\begin{array}{l}\text { Patients }< \\
\text { Controls }\end{array}$ & Precuneus & Left & $31 / 7$ & $0,-54,36$ & 4.49 & 69 & 0.004 \\
\hline $\begin{array}{l}\text { ReHo } \\
\text { a }\end{array}$ & $\begin{array}{l}\text { Patients }< \\
\text { Controls }\end{array}$ & Precuneus & Left & $31 / 7$ & $0,-52,36$ & 5.29 & 106 & 0.135 \\
\hline DC & $\begin{array}{l}\text { Patients } \\
\text { Controls }\end{array}$ & Precuneus & Left & $31 / 7$ & $-10,-58,36$ & 4.50 & 187 & 0.017 \\
\hline
\end{tabular}

\subsection{Seed-based Rsfc Analysis}

The rsFC map within each group is also shown in Fig. 2-A. Using the overlapped 47 voxels found above as the seed, we found that the BD group showed decreased rsFC of the seed with the left precuneus and the MPFC and increased rsFC (i.e., the decreased negative rsFC) of the seed with the left putamen (extended to the left insula) (Fig. 2-B and Table 3). 
Table 3

Between-group differences in the rsFC analyses between the patients with BD and the healthy controls

\begin{tabular}{|c|c|c|c|c|c|c|c|c|}
\hline Seeds & & Brain regions & Hemisphere & BA & $\begin{array}{l}\text { MNI } \\
\text { coordinates }\end{array}$ & $\begin{array}{l}\text { Peak } \\
\text { T } \\
\text { values }\end{array}$ & $\begin{array}{l}\text { Cluster } \\
\text { size }\end{array}$ & $\begin{array}{l}\text { Cluster- } \\
\text { level } \\
P_{\text {FWE }}\end{array}$ \\
\hline \multirow{3}{*}{$\begin{array}{l}47 \text { voxels in } \\
\text { the } \\
\text { precuneus } \\
\text { overlapped } \\
\text { across } \\
\text { fALFF, ReHo } \\
\text { and DC }\end{array}$} & $\begin{array}{l}\text { Patients } \\
> \\
\text { Controls }\end{array}$ & Putamen/insula & Left & $47 / 13$ & $-32,18,0$ & 4.92 & 217 & $<0.001$ \\
\hline & $\begin{array}{l}\text { Patients } \\
< \\
\text { Controls }\end{array}$ & Precuneus & Left/Right & $31 / 7$ & $-2,-50,36$ & 5.29 & 97 & 0.014 \\
\hline & & $\begin{array}{l}\text { Medial } \\
\text { prefrontal } \\
\text { cortex }\end{array}$ & Left/Right & 11 & $2,52,-14$ & 3.97 & 89 & 0.023 \\
\hline \multirow{3}{*}{$\begin{array}{l}57 \text { voxels in } \\
\text { the } \\
\text { precuneus } \\
\text { overlapped } \\
\text { between } \\
\text { fALFF and } \\
\text { DC }\end{array}$} & $\begin{array}{l}\text { Patients } \\
> \\
\text { Controls }\end{array}$ & Putamen/insula & Left & $47 / 13$ & $-32,18,0$ & 4.96 & 197 & $<0.001$ \\
\hline & $\begin{array}{l}\text { Patients } \\
< \\
\text { Controls }\end{array}$ & Precuneus & Left/Right & $31 / 7$ & $-2,-50,36$ & 5.50 & 109 & 0.006 \\
\hline & & $\begin{array}{l}\text { Medial } \\
\text { prefrontal } \\
\text { cortex }\end{array}$ & Left/Right & 11 & $2,52,-14$ & 4.03 & 89 & 0.023 \\
\hline $\begin{array}{l}\text { Precuneus } \\
\text { in the } \\
\text { Brainnetome } \\
\text { region } \\
\text { (No. } 153 \text { + } \\
\text { 154) }\end{array}$ & $\begin{array}{l}\text { Patients } \\
< \\
\text { Controls }\end{array}$ & $\begin{array}{l}\text { Medial } \\
\text { prefrontal } \\
\text { cortex }\end{array}$ & Left/Right & 11 & $2,52,-14$ & 4.50 & 126 & 0.014 \\
\hline
\end{tabular}

\subsection{Confirmation Analyses Of The Rsfc}

For the first confirmation analysis, when we used the significant region found in the fALFF and the DC analysis to identify the overlapped region, we found a cluster with 57 voxels located in the left precuneus (Fig. 3). Using this cluster as a seed, the rsFC map within each group is shown in Fig. 4-A. We found significantly decreased rsFC of the seed with the left precuneus and the MPFC and increased rsFC (i.e., the decreased negative rsFC) of the seed with the left putamen (Fig. 4-B and Table 3).

For the second confirmation analysis, we found that the significant clusters of every single functional index (i.e., fALFF, ReHo, and DC) and the overlapping clusters corresponded consistently to the brain regions No. 153 and 154, also named bilateral area 31 (A31) of precuneus, in the Human Brainnetome atlas (Fig. 5-A). Then, we used the sum of the brain region Nos. 153 and 154 as a seed in the following rsFC analysis. The rsFC map within each group is shown in 
Fig. 5-B. The whole-brain analysis found a significantly decreased rsFC of the seed with the MPFC (Fig. 5-C and Table 3).

\section{Discussion}

We used three main functional indices (fALFF, ReHo, and DC) to measure the spontaneous activity from the local activity, local connectivity to network-level functional connectivity. We found that the different functional indices convergently identified the decreased spontaneous activity in the left precuneus, a core region in the DMN, in patients with BD. Furthermore, using this overlapped region in the left precuneus as a seed for rsFC, we found the decreased rsFC within the DMN, indicated by the decreased rsFC with the left precuneus and decreased rsFC between the seed precuneus and the MPFC in patients with BD. In addition, we found decreased negative functional connectivity between the left precuneus and the core regions in the SN (i.e., the left putamen/insula), suggesting decreased anticorrelation between the DMN and the SN in patients with $\mathrm{BD}$.

\subsection{Altered Spontaneous Activity Of The Dmn}

We used multiple functional indices and found that the patients with BD exhibited decreased fAlff, ReHo, and DC values in the left precuneus (a specific brain region of the DMN) compared with healthy controls. The decrease in the fALFF indicates reduced local BOLD signal fluctuations of the precuneus ${ }^{22,39}{ }^{3}$. The decrease in the ReHo indicates decreased synchronizing ability in relevant voxels within the precuneus ${ }^{23}$, and the decrease in DC indicates reduced functional connections of the precuneus with other voxels in the brain ${ }^{40}$. Our findings are consistent with most previous studies $^{26,41,42}$, which found decreased spontaneous brain activities of the left precuneus in patients with BD. However, another study, which only recruited 17 bipolar depression patients and $16 \mathrm{HCs}$, reported increased ReHo values of the left precuneus in patients ${ }^{43}$.

The dysfunction detected by these functional indices may be explained by the abnormal metabolism and structure in this region. One PET study found reduced metabolism of the left precuneus in the old euthymic patients with BD ${ }^{44}$. Another study using cortical gray matter thickness as an indicator found cortical thinning of the left precuneus in 1837 patients with $\mathrm{BD}{ }^{45}$. The precuneus is located in the posterior medial part of the parietal lobe, which is extensively connected with cortex and subcortical brain areas ${ }^{46}$. The precuneus is associated with emotion regulation ${ }^{47}$, and is especially sensitive to negative emotion. A left lateralized activation of the precuneus was found when participants viewed negative images ${ }^{48}$ or when they were asked to increase their negative emotions when facing aversive stimuli ${ }^{49}$. One study using a negative emotion task found reduced activation of the left precuneus during negative images condition in the euthymic BD-I patients, with respect to controls ${ }^{50}$. This finding suggested that decreased brain activity of the left precuneus in patients with BD may somehow interfere with their ability to process emotional contents. The results found in the current resting-state fMRI study highlighted the importance of the precuneus, especially its left part, in the pathogenesis of BD. Thus, the patients' left precuneus was unable to maintain normal signal fluctuation, synchronizing ability, and connectivity with other brain regions.

\subsection{Decreased Rsfc Within The Dmn}

The followed rsFC analysis found hypoconnectivity within the left precuneus in the patients with BD. The results were consistent with previous studies ${ }^{51,52}$. Wang et al. ${ }^{51}$ used FC strength (FCS) to measure the whole-brain FCS patterns, and found that unmedicated patients with BD-II depression showed decreased FCS in the DMN (including the left 
precuneus). Khadka et al. ${ }^{52}$ using ICA found that the rsFC in the posterior DMN consisting mainly of bilateral precuneus was significantly decreased in patients with psychotic BD-I than the HC. Combining these findings with our results, we speculated that the decreased local spontaneous activity of the left precuneus may account for its decreased rsFC. In addition, the precuneus is a functional core of the $\mathrm{DMN}^{53}$. It not only shows the highest resting metabolic rate within the network ${ }^{54}$, but has widespread connectivity with other brain regions ${ }^{46}$. It suggested that the anomaly of precuneus could have an important effect on the overall functional abnormality of the DMN 46,53 .

The patients with BD also exhibited reduced rsFC between the left precuneus and the MPFC, which is another core node of the $\mathrm{DMN}^{55}$. This result was repeated in our confirmation analyses. The current result was, to some extent, consistent with previous report of Wang et al. ${ }^{56}$, although two additional studies using smaller sample size (the number of patients with BD is 15 and 30 ) did not find positive results ${ }^{21,57}$. In Wang's study, unmedicated patients with BD under depressive episode were included, and patients showed disrupted intramodular connectivity within the DMN. The reason for the hypoconnectivity within the DMN may be the dysfunction of neurotransmission, such as one study found that the rsFC of the core DMN (composed of the precuneus and the MPFC) is related to $\mathrm{K}+$ transport protein, axonal guidance protein, neuronal generation/development protein, and cell differentiation protein ${ }^{58,59}$. The reduced rsFC between the MPFC and the left precuneus reflected weaker synchronization of spontaneous neural activities within the DMN, which could reduce the efficiency of the information communication and integration of the network ${ }^{60}$. The precuneus is involved in self-centered mental imagery strategies and episodic memory retrieval ${ }^{46}$. The MPFC is one of the most clearly delineated region in terms of its functional roles; it serves an important role in emotional experience, social cognition, memory, and decision making ${ }^{61,62}$. Some researchers suggested that the abnormal rsFC between the anterior (e.g., the MPFC and the superior frontal gyrus) and the posterior (e.g., the PCC/precuneus) components of the DMN sever as the neural basis for the defects of attention and working memory ${ }^{11}$. The patients with BD have been reported to have these cognitive abnormalities ${ }^{63}$. One study on the relationship between the DMN and clinical symptoms of BD found that the decreased rsFC within the posterior DMN in patients with BD depression might be associated with rumination ${ }^{16}$, which is a typical symptom for depressed patients. It is characterized as a pattern of response to pain or stress that involves repetition and passive attention to negative self-related information ${ }^{64}$. From this aspect, it would be reasonable to speculate that the decreased rsFC between the MPFC and the precuneus may result in the cognitive dysfunction and clinical symptoms for patients with BD. In summary, our findings with all previous studies support that the functional dysconnectivity within the DMN may play a relatively important role in the neural basis of BD.

\subsection{Decreased Anticorrelation Between Dmn And Sn}

Decreased negative connectivity was found between the left precuneus and the left putamen/insula, the main nodes of the $\mathrm{SN}^{2,65}$, in the patients with $\mathrm{BD}$. This finding reflects the decreased anticorrelation between the DMN and the SN.

In healthy population, resting-state fMRI signals of the DMN are anticorrelated with that of task-positive networks, such as the $\mathrm{SN}^{66-68}$. The SN generates control signals and causally mediates the "switching" between the DMN and the central executive network ${ }^{69}$. This condition leads to a switch between their functions of introspective and extroversive attention orientation, and then allows individuals to stay vigilant when meeting unexpected environmental events ${ }^{11}$. This anticorrelation between DMN and SN has even been proven more important than the DMN's own activity ${ }^{70}$. The decreased anticorrelation between these networks may result in the reduction of introspective thinking and attention deficit, which could be the potential interference source of goal-directed behavior ${ }^{11}$. Weaker anticorrelation between the DMN and the $\mathrm{SN}$ was also found in many other brain disorders ${ }^{66}$. Our result expanded previous findings of disruption 
of the DMN-SN functional connectivity in mental disorders ${ }^{20}$ by showing that the negative rsFC between the left precuneus and the left putamen/insula was decreased in patients with BD.

\section{Limitations}

Some limitations are as follows. First, although the patients were not in a manic mood state, our sample was still heterogeneous in the subtypes or the stages of the illness. Thus, future work should recruit patients in the same mood state (mania, depression, or euthymia) to identify whether the significant results were state-dependent or trait-like characters for BD. Second, most patients have taken different types or dosages of drugs. Although no consistent effect of a certain drug was found on BOLD signals, different drugs or dosages may influence BOLD signals from different aspects $^{71}$, thereby interfering our results. In the future, unmedicated patients with BD should be recruited or the types and dosages of the patients' drug use should be strictly controlled. Third, our study did not consider whether these changes in the brain could account for any clinical or cognitive symptoms, such as rumination, and whether these brain abnormalities have changed over time. Thus, further studies are necessary to address these issues.

\section{Conclusions}

In summary, by using multiple functional indices, we found the decreased spontaneous brain activity in DMN, particularly located in the left precuneus, and decreased functional connectivity within the DMN and the disruptive anticorrelation between the DMN and the SN in patients with BD. These findings suggest that the DMN is a key aspect for understanding the neural basis of $\mathrm{BD}$, and the altered functional patterns of $\mathrm{DMN}$ may be a potential candidate biomarker for diagnosis of BD.

\section{Abbreviations}

BD, bipolar disorder; rs-fMRI, resting-state functional magnetic resonance imaging; DMN, default mode network; MPFC, medial prefrontal cortex; PCC, posterior cingulate cortex; SN, salience network; fALFF, fractional amplitude of lowfrequency fluctuation; ReHo, regional homogeneity, DC, degree centrality; ICA, independent component analysis; HAMD, Hamilton Depression Scale; HC, healthy control; YMRS, Young Mania Rating Scale.

\section{Declarations}

\section{Acknowledgments}

None

Funding Supported by National Science and Technology Major Project for IND (2018ZX09201-014), Beijing Municipal Administration of Hospitals Clinical Medicine Development of Special Funding (ZYLX201807), Beijing Municipal Natural Science Foundation (7192081) and National Natural Science Foundation of China (81901355).

\section{Conflicts of interest None}

Ethical approval All procedures performed in studies involving human participants were in accordance with the ethical standards of the institutional and/or national research committee and with the 1964 Helsinki declaration and its later amendments or comparable ethical standards.

Informed consent Informed consent was obtained from all individual participants included in the study. 
Availability of data and material Data available on request from the authors.

\section{Author contributions}

conceptualization: Y.Z C.W, methodology: L.Z Z.Z, analysis: L.Z Z.Z, resources: F.L Q.B, writing (original draft preparation): L.Z Q.B, writing (review and editing): Y.Z C.W, supervision: C.W, project administration: C.W, funding acquisition: C.W Q.B

\section{References}

1. Grande, I., Berk, M., Birmaher, B., \& Vieta, E. Bipolar disorder. Lancet (London, England) 2016 Apr 9;387(10027):1561-1572.

2. Huang, Y., Wang, Y., Wang, H., et al. (2019 Mar). Prevalence of mental disorders in China: a cross-sectional epidemiological study. The lancet Psychiatry, 6(3), 211-224.

3. Phillips, M. L., \& Swartz, H. A. (2014 Aug). A critical appraisal of neuroimaging studies of bipolar disorder: toward a new conceptualization of underlying neural circuitry and a road map for future research. The American journal of psychiatry, 171(8), 829-843.

4. Townsend, J., \& Altshuler, L. L. (2012 Jun). Emotion processing and regulation in bipolar disorder: a review. Bipolar disorders, 14(4), 326-339.

5. Vargas, C., Lopez-Jaramillo, C., \& Vieta, E. A systematic literature review of resting state network-functional MRI in bipolar disorder. Journal of affective disorders 2013 Sep 25;150(3):727-735.

6. Raichle, M. E. The brain's default mode network. Annual review of neuroscience 2015 Jul 8;38:433-447.

7. Buckner, R. L., Andrews-Hanna, J. R., \& Schacter, D. L. The brain's default network: anatomy, function, and relevance to disease. Annals of the New York Academy of Sciences 2008 Mar;1124:1-38.

8. Greicius, M. D., Krasnow, B., Reiss, A. L., \& Menon, V. Functional connectivity in the resting brain: a network analysis of the default mode hypothesis. Proceedings of the National Academy of Sciences of the United States of America 2003 Jan 7;100(1):253-258.

9. Raichle, M. E., MacLeod, A. M., Snyder, A. Z., Powers, W. J., Gusnard, D. A., \& Shulman, G. L. A default mode of brain function. Proceedings of the National Academy of Sciences of the United States of America 2001 Jan 16;98(2):676-682.

10. Zovetti, N., Rossetti, M. G., Perlini, C., et al. Default mode network activity in bipolar disorder. Epidemiology and psychiatric sciences 2020 Sep 8;29:e166.

11. Broyd, S. J., Demanuele, C., Debener, S., Helps, S. K., James, C. J., \& Sonuga-Barke, E. J. S. Default-mode brain dysfunction in mental disorders: A systematic review. Neuroscience \& Biobehavioral Reviews 2009 2009/03/01/;33(3):279-296.

12. Martino, M., Magioncalda, P., Huang, Z., et al. Contrasting variability patterns in the default mode and sensorimotor networks balance in bipolar depression and mania. Proceedings of the National Academy of Sciences of the United States of America 2016 Apr 26;113(17):4824-4829.

13. Wang, Y., Gao, Y., Tang, S., et al. (2020 Apr). Large-scale network dysfunction in the acute state compared to the remitted state of bipolar disorder: A meta-analysis of resting-state functional connectivity. EBioMedicine, 54, 102742.

14. Bellani, M., Bontempi, P., Zovetti, N., et al. Resting state networks activity in euthymic Bipolar disorder. Bipolar disorders 2020 Mar 25. 
15. Chen, G., Zhao, L., Jia, Y., et al. Abnormal cerebellum-DMN regions connectivity in unmedicated bipolar II disorder. Journal of affective disorders 2019 Jan 15;243:441-447.

16. Gong, J., Chen, G., Jia, Y., et al. Disrupted functional connectivity within the default mode network and salience network in unmedicated bipolar II disorder. Progress in neuro-psychopharmacology \& biological psychiatry 2019 Jan 10;88:11-18.

17. Liu, Y., Wu, X., Zhang, J., Guo, X., Long, Z., \& Yao, L. Altered effective connectivity model in the default mode network between bipolar and unipolar depression based on resting-state fMRI. Journal of affective disorders 2015 Aug 15;182:8-17.

18. Ongür, D., Lundy, M., Greenhouse, I., et al. Default mode network abnormalities in bipolar disorder and schizophrenia. Psychiatry research 2010 Jul 30;183(1):59-68.

19. Wang, J., Wang, Y., Wu, X., et al. (2020 Feb). Shared and specific functional connectivity alterations in unmedicated bipolar and major depressive disorders based on the triple-network model. Brain imaging and behavior, 14(1), 186199.

20. Magioncalda, P., Martino, M., Conio, B., et al. (2015 Feb). Functional connectivity and neuronal variability of resting state activity in bipolar disorder-reduction and decoupling in anterior cortical midline structures. Human brain mapping, 36(2), 666-682.

21. Nguyen, T. T., Kovacevic, S., Dev, S. I., Lu, K., Liu, T. T., \& Eyler, L. T. (2017 Jan). Dynamic functional connectivity in bipolar disorder is associated with executive function and processing speed: A preliminary study. Neuropsychology, 37(1), 73-83.

22. Zou, Q. H., Zhu, C. Z., Yang, Y., et al. An improved approach to detection of amplitude of low-frequency fluctuation (ALFF) for resting-state fMRI: fractional ALFF. Journal of neuroscience methods 2008 Jul 15;172(1):137-141.

23. Zang, Y., Jiang, T., Lu, Y., He, Y., \& Tian, L. (2004 May). Regional homogeneity approach to fMRI data analysis. Neurolmage, 22(1), 394-400.

24. Buckner, R. L., Sepulcre, J., Talukdar, T., et al. Cortical hubs revealed by intrinsic functional connectivity: mapping, assessment of stability, and relation to Alzheimer's disease. The Journal of neuroscience: the official journal of the Society for Neuroscience 2009 Feb 11;29(6):1860-1873.

25. Liu, C. H., Ma, X., Li, F., et al. (2012). Regional homogeneity within the default mode network in bipolar depression: a resting-state functional magnetic resonance imaging study. PloS one, 7(11), e48181.

26. Qiu, M., Zhang, H., Mellor, D., et al. (2018). Aberrant Neural Activity in Patients With Bipolar Depressive Disorder Distinguishing to the Unipolar Depressive Disorder: A Resting-State Functional Magnetic Resonance Imaging Study. Frontiers in psychiatry, 9, 238.

27. Yang, Y., Liu, S., Jiang, X., et al. (2019). Common and Specific Functional Activity Features in Schizophrenia, Major Depressive Disorder, and Bipolar Disorder. Frontiers in psychiatry, 10, 52.

28. Russo, D., Martino, M., Magioncalda, P., Inglese, M., Amore, M., \& Northoff, G. Opposing Changes in the Functional Architecture of Large-Scale Networks in Bipolar Mania and Depression. Schizophrenia bulletin 2020 Feb 12.

29. Zhang, Z., Bo, Q., Li, F., et al. Increased ALFF and functional connectivity of the right striatum in bipolar disorder patients. Progress in neuro-psychopharmacology \& biological psychiatry 2020 Oct 14:110140.

30. First, M. B., Spitzer, R. L., Gibbon, M., Williams, J. B. W., Fei, L. P., \& Liu, X. H. (Eds.). (2002). Structured Clinical Interview for DSM-IV-TR Axis I Disorders - Patient Edition (SCID- I/P): Biometrics Research. New York: State Psychiatric Institute, New York.

31. Young, R. C., Biggs, J. T., Ziegler, V. E., \& Meyer, D. A. A rating scale for mania: reliability, validity and sensitivity. The British journal of psychiatry: the journal of mental science 1978 Nov;133:429-435. 
32. Hamilton, M. (1967 Dec). Development of a rating scale for primary depressive illness. Br J Soc Clin Psychol, 6(4), 278-296.

33. Yan, C.-G., Wang, X.-D., Zuo, X.-N., \& Zang, Y.-F. J. N. DPABI: data processing \& analysis for (resting-state) brain imaging. 2016;14(3):339-351.

34. Friston, K. J., Williams, S., Howard, R., Frackowiak, R. S., \& Turner, R. (1996 Mar). Movement-related effects in fMRI time-series. Magn Reson Med, 35(3), 346-355.

35. Power, J. D., Barnes, K. A., Snyder, A. Z., Schlaggar, B. L., \& Petersen, S. E. Spurious but systematic correlations in functional connectivity MRI networks arise from subject motion. Neuroimage 2012 Feb 1;59(3):2142-2154.

36. Power, J. D., Mitra, A., Laumann, T. O., Snyder, A. Z., Schlaggar, B. L., \& Petersen, S. E. Methods to detect, characterize, and remove motion artifact in resting state fMRI. Neurolmage 2014 Jan 1;84:320-341.

37. Zuo, X. N., Ehmke, R., Mennes, M., et al. (2012 Aug). Network centrality in the human functional connectome. Cereb Cortex, 22(8), 1862-1875.

38. Fan, L., Li, H., Zhuo, J., et al. The Human Brainnetome Atlas: A New Brain Atlas Based on Connectional Architecture. Cerebral cortex (New York, NY: 1991) 2016 Aug;26(8):3508-3526.

39. Wang, J. J., Chen, X., Sah, S. K., et al. (2016 Jun). Amplitude of low-frequency fluctuation (ALFF) and fractional ALFF in migraine patients: a resting-state functional MRI study. Clinical radiology, 71(6), 558-564.

40. Wang, X., Jiao, D., Zhang, X., \& Lin, X. Altered degree centrality in childhood absence epilepsy: A resting-state fMRI study. Journal of the neurological sciences 2017 Feb 15;373:274-279.

41. Lu, D., Jiao, Q., Zhong, Y., et al. (2014). Altered baseline brain activity in children with bipolar disorder during mania state: a resting-state study. Neuropsychiatric disease and treatment, 10, 317-323.

42. Jiang, X., Fu, S., Yin, Z., et al. Common and distinct neural activities in frontoparietal network in first-episode bipolar disorder and major depressive disorder: Preliminary findings from a follow-up resting state fMRI study. Journal of affective disorders 2020 Jan 1;260:653-659.

43. Liang, M. J., Zhou, Q., Yang, K. R., et al. (2013). Identify changes of brain regional homogeneity in bipolar disorder and unipolar depression using resting-state FMRI. PloS one, 8(12), e79999.

44. Brooks, J. O. 3rd, Hoblyn, J. C., Woodard, S. A., Rosen, A. C., \& Ketter, T. A. (2009 Feb). Corticolimbic metabolic dysregulation in euthymic older adults with bipolar disorder. Journal of psychiatric research, 43(5), 497-502.

45. Hibar, D. P., Westlye, L. T., Doan, N. T., et al. (2018 Apr). Cortical abnormalities in bipolar disorder: an MRI analysis of 6503 individuals from the ENIGMA Bipolar Disorder Working Group. Molecular psychiatry, 23(4), 932-942.

46. Cavanna, A. E., \& Trimble, M. R. (2006 Mar). The precuneus: a review of its functional anatomy and behavioural correlates. Brain: a journal of neurology, 129(Pt 3), 564-583.

47. Wegbreit, E., Cushman, G. K., Puzia, M. E., et al. (2014 Aug). Developmental meta-analyses of the functional neural correlates of bipolar disorder. JAMA psychiatry, 71(8), 926-935.

48. Meseguer, V., Romero, M. J., Barrós-Loscertales, A., et al. (2007 Aug). Mapping the apetitive and aversive systems with emotional pictures using a block-design fMRI procedure. Psicothema, 19(3), 483-488.

49. Ochsner, K. N., Ray, R. D., Cooper, J. C., et al. (2004 Oct). For better or for worse: neural systems supporting the cognitive down- and up-regulation of negative emotion. Neurolmage, 23(2), 483-499.

50. Sepede, G., De Berardis, D., Campanella, D., et al. Neural correlates of negative emotion processing in bipolar disorder. Progress in neuro-psychopharmacology \& biological psychiatry $2015 \mathrm{Jul}$ 3;60:1-10.

51. Wang, Y., Zhong, S., Jia, Y., et al. (2016 Aug). Disrupted Resting-State Functional Connectivity in Nonmedicated Bipolar Disorder. Radiology, 280(2), 529-536. 
52. Khadka, S., Meda, S. A., Stevens, M. C., et al. Is aberrant functional connectivity a psychosis endophenotype? A resting state functional magnetic resonance imaging study. Biological psychiatry 2013 Sep 15;74(6):458-466.

53. Utevsky, A. V., Smith, D. V., \& Huettel, S. A. Precuneus is a functional core of the default-mode network. The Journal of neuroscience: the official journal of the Society for Neuroscience 2014 Jan 15;34(3):932-940.

54. Gusnard, D. A., Raichle, M. E., \& Raichle, M. E. (2001 Oct). Searching for a baseline: functional imaging and the resting human brain. Nature reviews Neuroscience, 2(10), 685-694.

55. Syan, S. K., Smith, M., Frey, B. N., et al. Resting-state functional connectivity in individuals with bipolar disorder during clinical remission: a systematic review. Journal of psychiatry \& neuroscience: JPN 2018 Aug;43(5):298316.

56. Wang, Y., Wang, J., Jia, Y., et al. Topologically convergent and divergent functional connectivity patterns in unmedicated unipolar depression and bipolar disorder. Translational psychiatry 2017 Jul 4;7(7):e1165.

57. Lois, G., Linke, J., \& Wessa, M. (2014). Altered functional connectivity between emotional and cognitive resting state networks in euthymic bipolar I disorder patients. PloS one, 9(10), e107829.

58. Meda, S. A., Ruaño, G., Windemuth, A., et al. Multivariate analysis reveals genetic associations of the resting default mode network in psychotic bipolar disorder and schizophrenia. Proceedings of the National Academy of Sciences of the United States of America 2014 May 13;111(19):E2066-2075.

59. Smolin, B., Karry, R., Gal-Ben-Ari, S., \& Ben-Shachar, D. (2012 Aug). Differential expression of genes encoding neuronal ion-channel subunits in major depression, bipolar disorder and schizophrenia: implications for pathophysiology. The international journal of neuropsychopharmacology, 15(7), 869-882.

60. Fingelkurts, A. A., Fingelkurts, A. A., \& Kähkönen, S. Functional connectivity in the brain-is it an elusive concept? Neuroscience and biobehavioral reviews 2005 Jan;28(8):827-836.

61. Lieberman, M. D., Straccia, M. A., Meyer, M. L., Du, M., \& Tan, K. M.. Social, self, (situational), and affective processes in medial prefrontal cortex (MPFC): Causal, multivariate, and reverse inference evidence. Neuroscience and biobehavioral reviews 2019 Apr;99:311-328.

62. Euston, D. R., Gruber, A. J., \& McNaughton, B. L. The role of medial prefrontal cortex in memory and decision making. Neuron 2012 Dec 20;76(6):1057-1070.

63. Alonso-Lana, S., Moro, N., McKenna, P. J., et al. (2019 Aug). Longitudinal brain functional changes between mania and euthymia in bipolar disorder. Bipolar disorders, 21(5), 449-457.

64. Nolen-Hoeksema, S., Wisco, B. E., \& Lyubomirsky, S. Rethinking Rumination. Perspectives on psychological science: a journal of the Association for Psychological Science 2008 Sep;3(5):400-424.

65. Palaniyappan, L., White, T. P., \& Liddle, P. F. (2012). The concept of salience network dysfunction in schizophrenia: from neuroimaging observations to therapeutic opportunities. Current topics in medicinal chemistry, 12(21), 23242338.

66. Muthulingam, J. A., Hansen, T. M., Drewes, A. M., Olesen, S. S., \& Frokjaer, J. B. Disrupted functional connectivity of default mode and salience networks in chronic pancreatitis patients. Clinical neurophysiology: official journal of the International Federation of Clinical Neurophysiology 2020 Feb 10;131(5):1021-1029.

67. Peng, D., Liddle, E. B., Iwabuchi, S. J., et al. Dissociated large-scale functional connectivity networks of the precuneus in medication-naive first-episode depression. Psychiatry research 2015 Jun 30;232(3):250-256.

68. Chai, X. J., Castanon, A. N., Ongur, D., \& Whitfield-Gabrieli, S. Anticorrelations in resting state networks without global signal regression. Neurolmage 2012 Jan 16;59(2):1420-1428.

69. Uddin, L. Q. (2015 Jan). Salience processing and insular cortical function and dysfunction. Nature reviews Neuroscience, 16(1), 55-61. 
70. Uddin, L. Q., Kelly, A. M., Biswal, B. B., Castellanos, F. X., \& Milham, M. P. (2009 Feb). Functional connectivity of default mode network components: correlation, anticorrelation, and causality. Human brain mapping, 30(2), 625637.

71. Hafeman, D. M., Chang, K. D., Garrett, A. S., Sanders, E. M., \& Phillips, M. L. (2012 Jun). Effects of medication on neuroimaging findings in bipolar disorder: an updated review. Bipolar disorders, 14(4), 375-410.

\section{Figures}

A
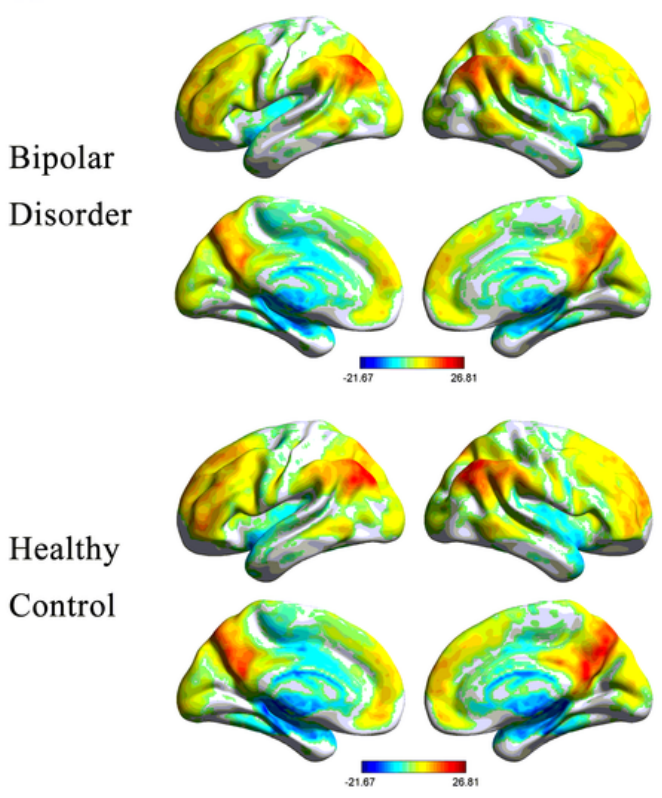

$\mathrm{ReHo}$
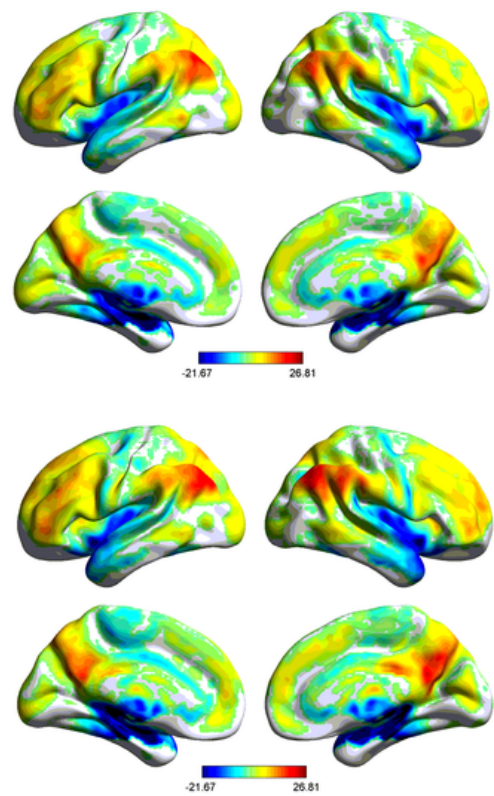

DC
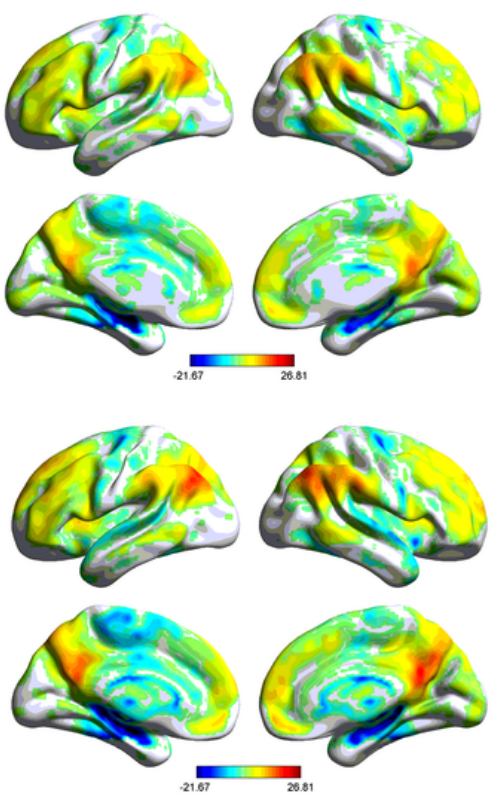

B
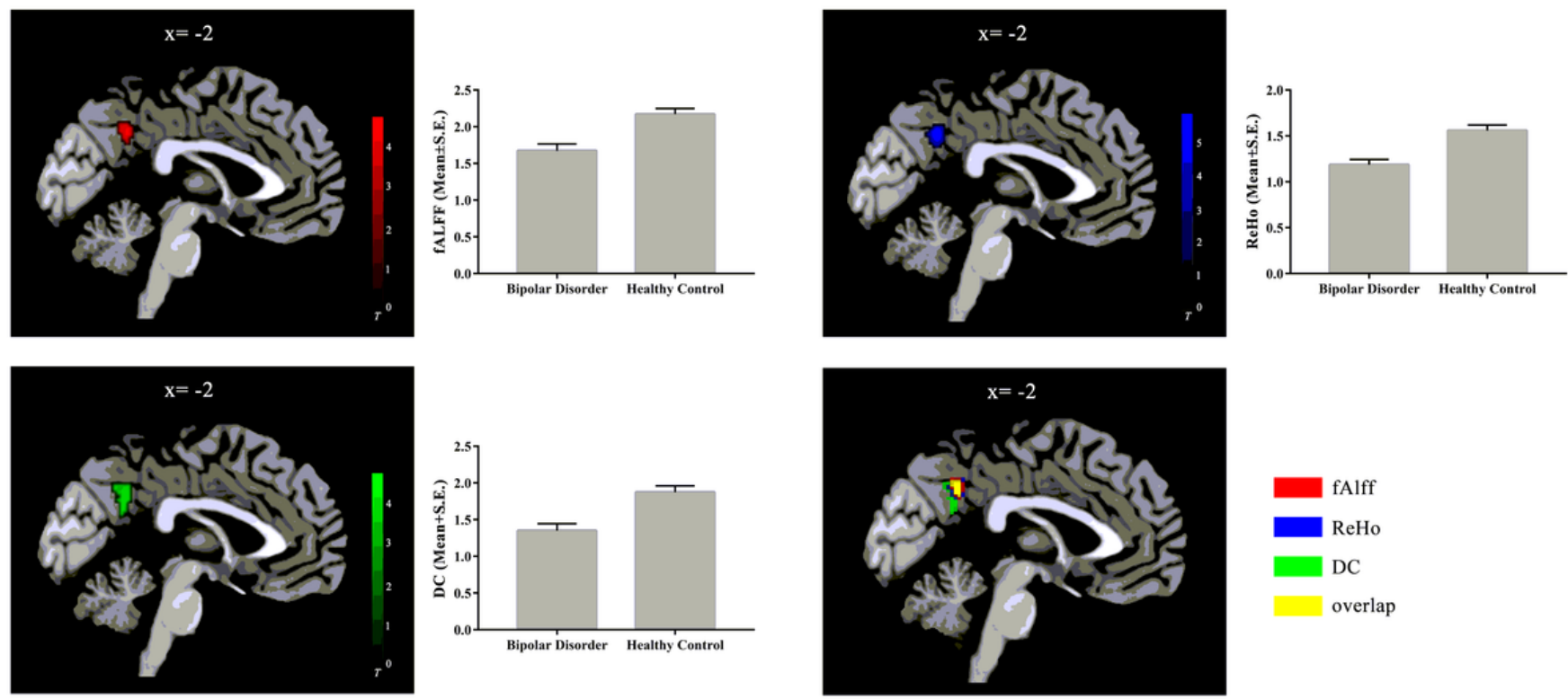

Figure 1

fAlff, ReHo, and DC maps within each group (A) and between-group differences in each functional index (B). In panel B, the patients with BD showed decreased fAlff (in red), ReHo (in blue), and DC (in green) in the left precuneus. The 
overlapped cluster (47 voxels) across these functional indices is represented in yellow.

A

Bipolar Disorder
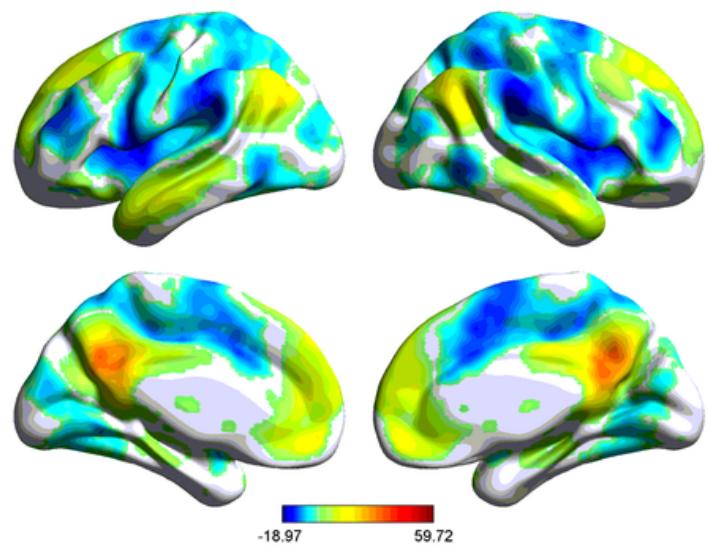

Healthy Control
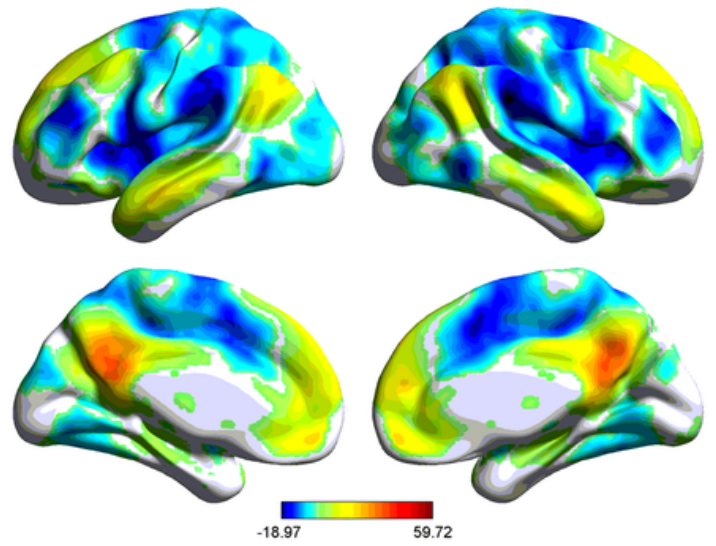

\section{B}
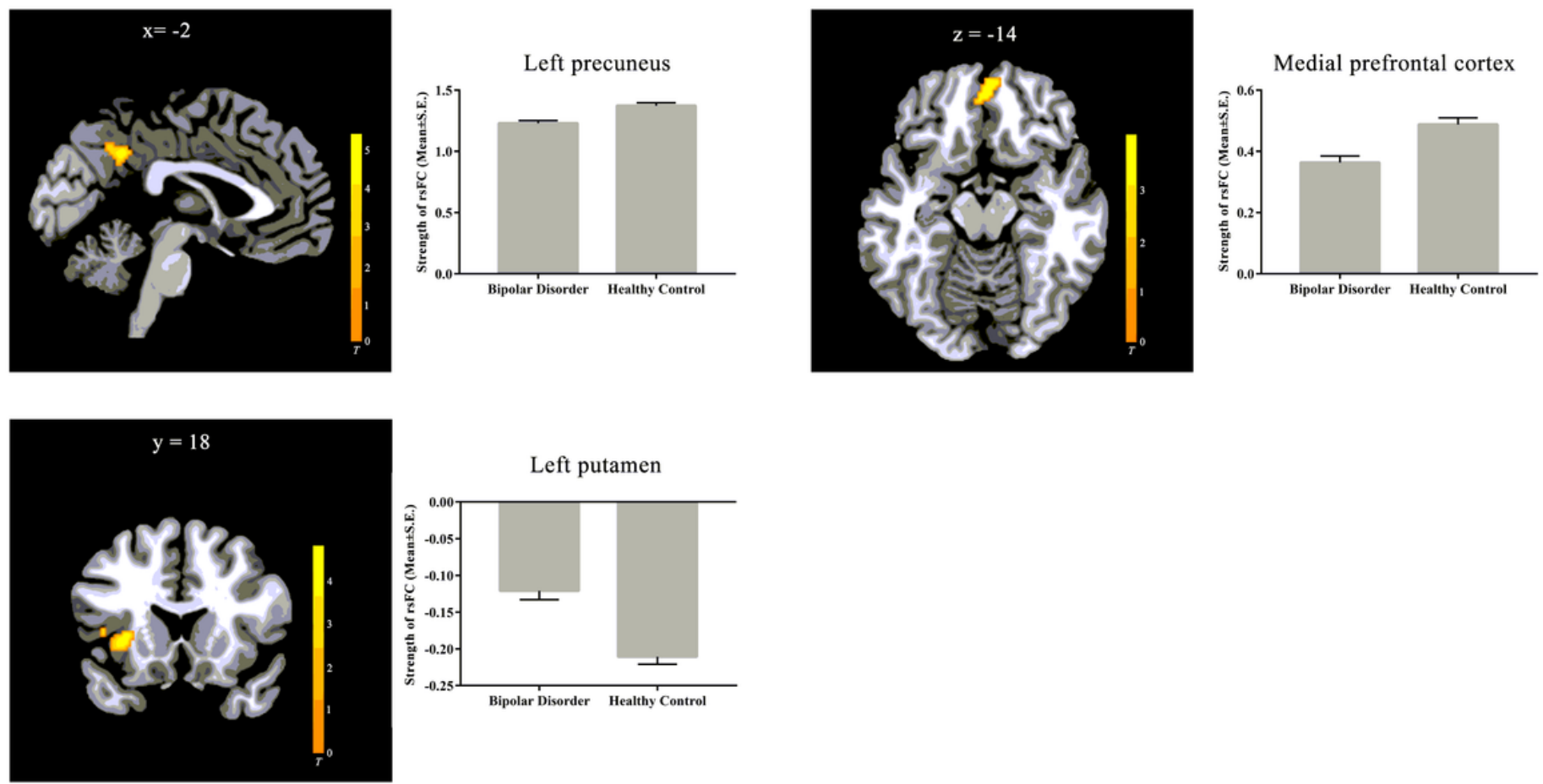

\section{Figure 2}

rsFC (using the overlapped 47 voxels as a seed) map within each group (A) and between-group differences in the rsFC map (B). The panel B showed that the left precuneus and the medial prefrontal cortex whose rsFC with the left precuneus were decreased, and the left putamen whose rsFC with the left precuneus was increased in patients with BD. 


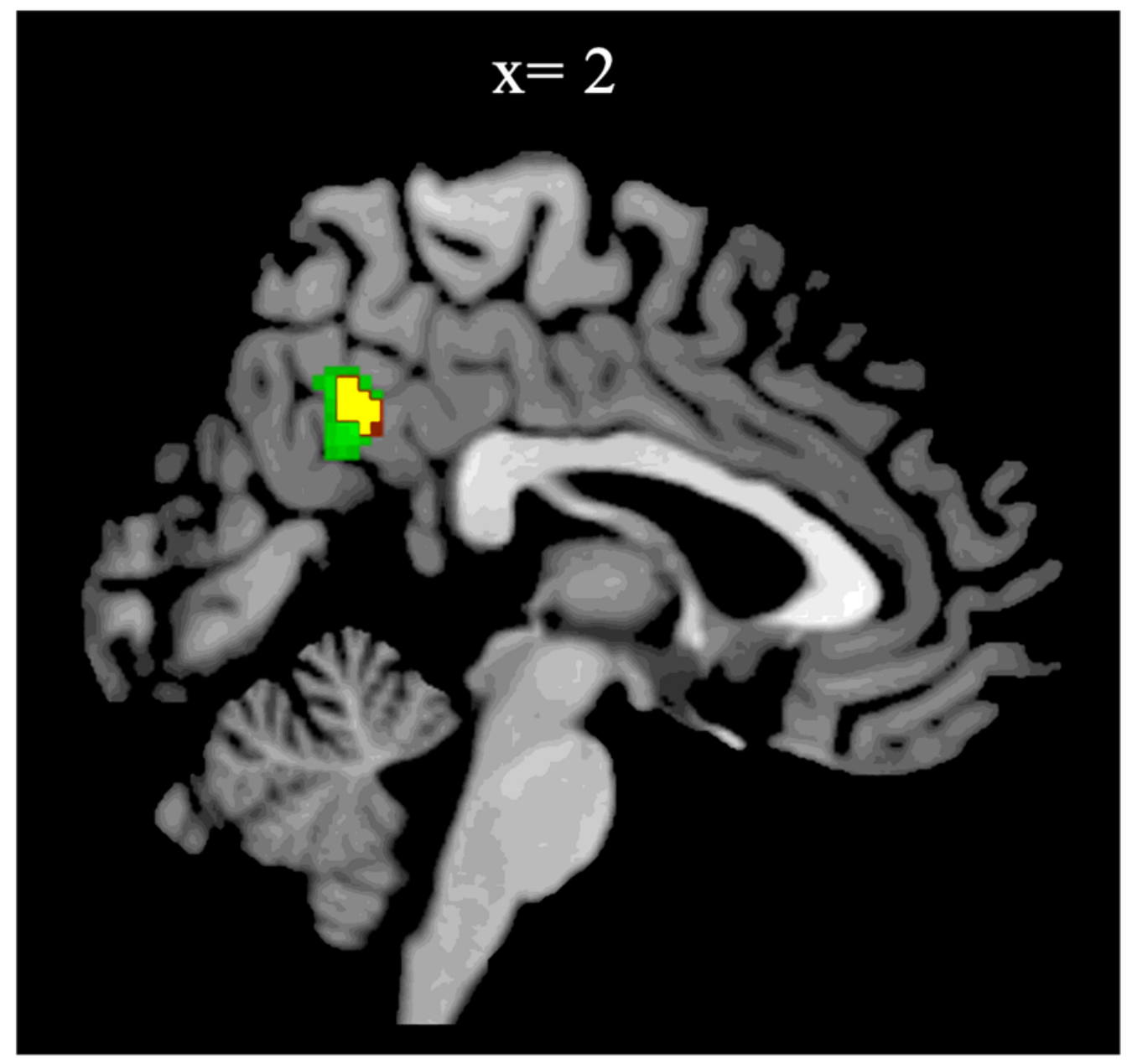

fAlff

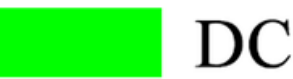

overlap

Figure 3

Overlapped cluster (57 voxels) between the fALFF and DC analyses. The patients with BD showed decreased fAlff (in red) and DC (in green) in the left precuneus. The overlapped cluster (57 voxels) was represented in yellow. 
A

Bipolar Disorder
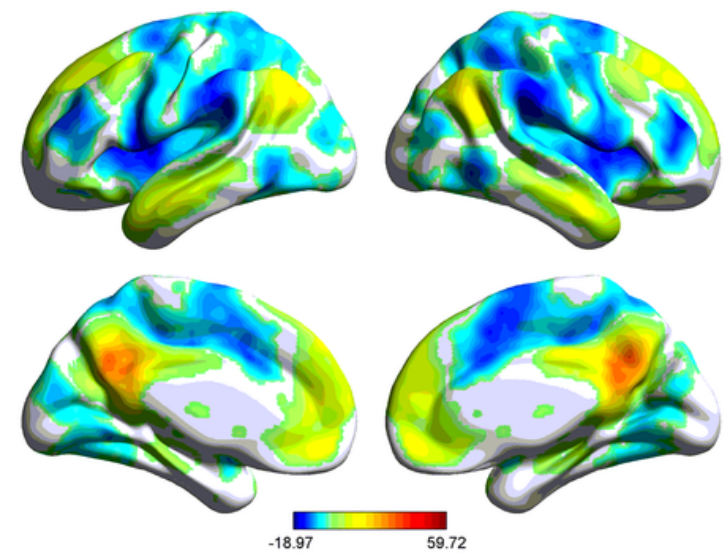

Healthy Control
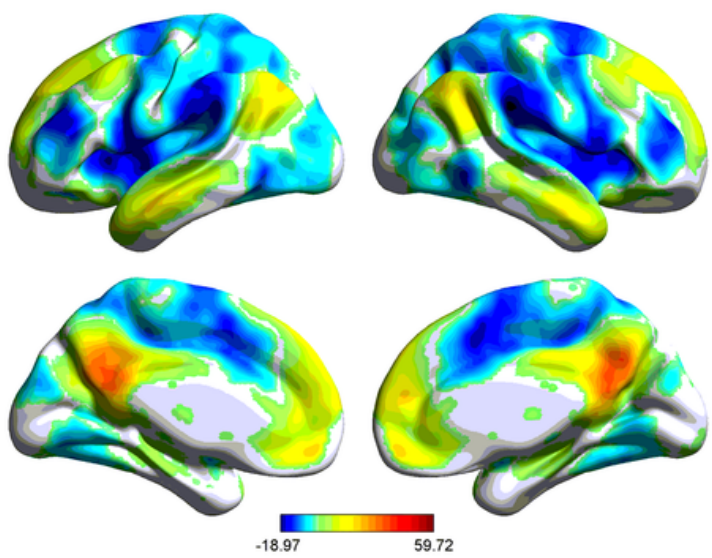

B
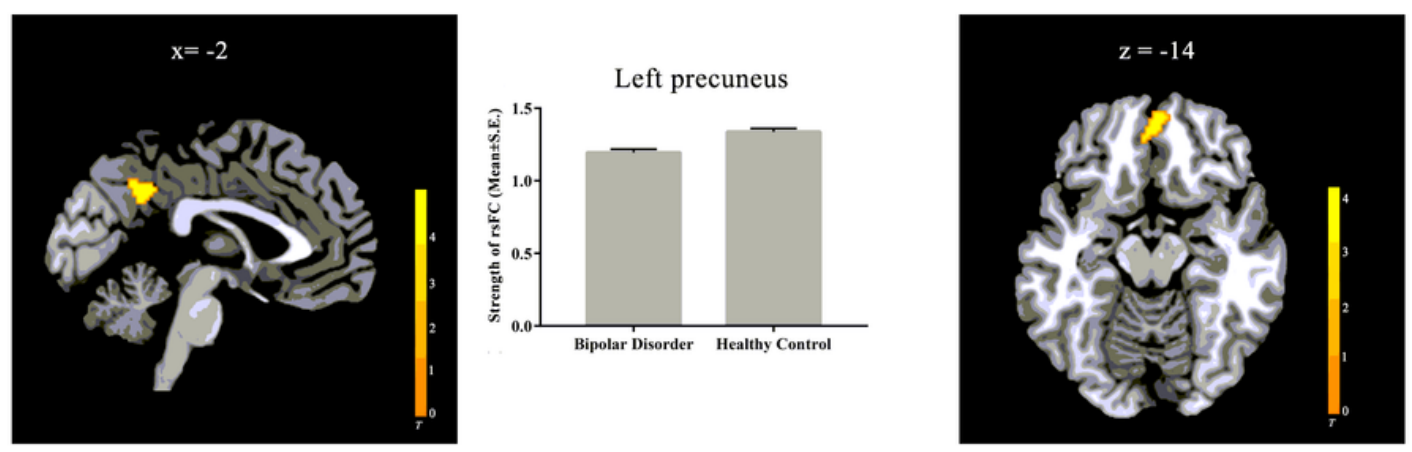

Medial prefrontal cortex
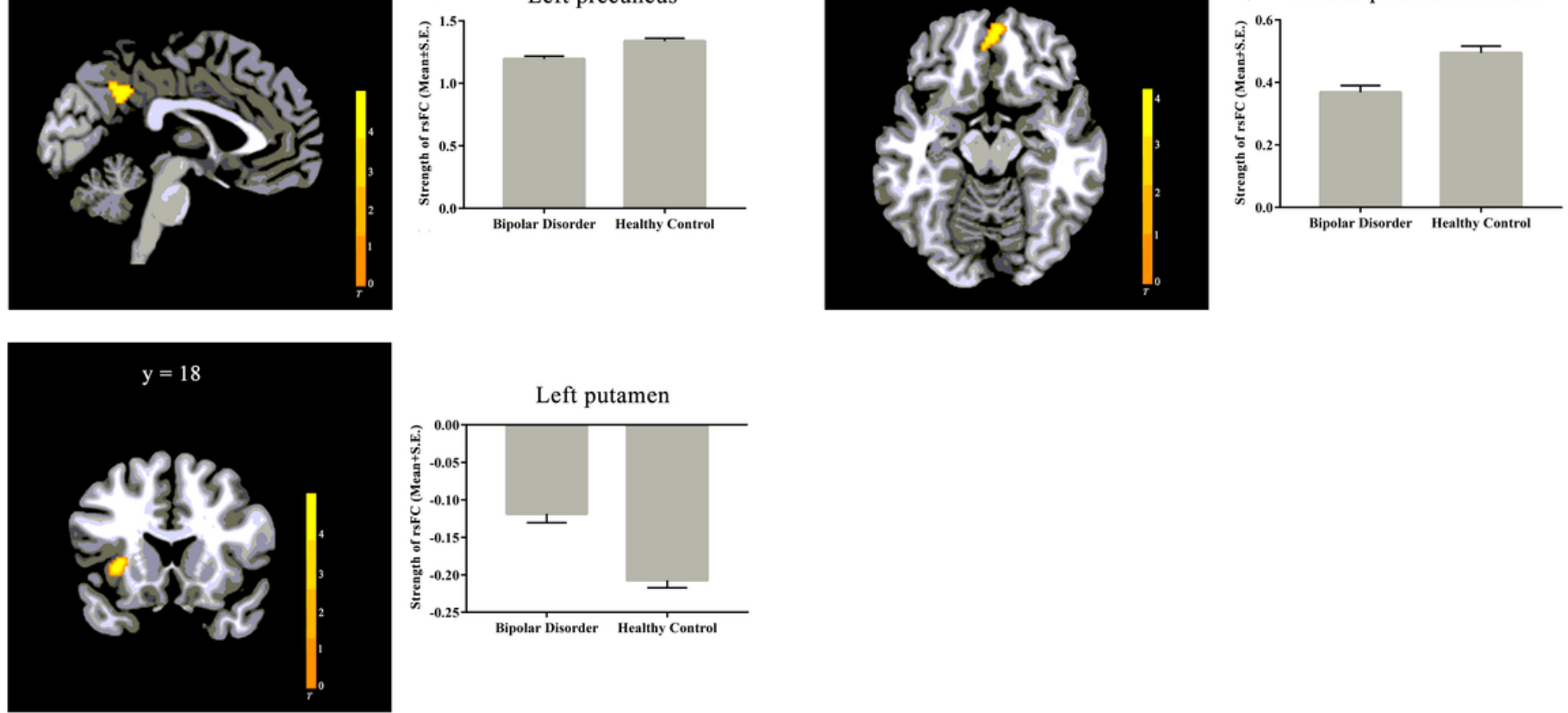

\section{Figure 4}

rsFC (using the overlapped 57 voxels as a seed) map within each group (A) and between-group differences in the rsFC map (B). The panel B showed that the left precuneus and the medial prefrontal cortex whose rsFC with the left precuneus were decreased, and the left putamen whose rsFC with the left precuneus was increased in patients with BD. 
$\mathbf{A}$
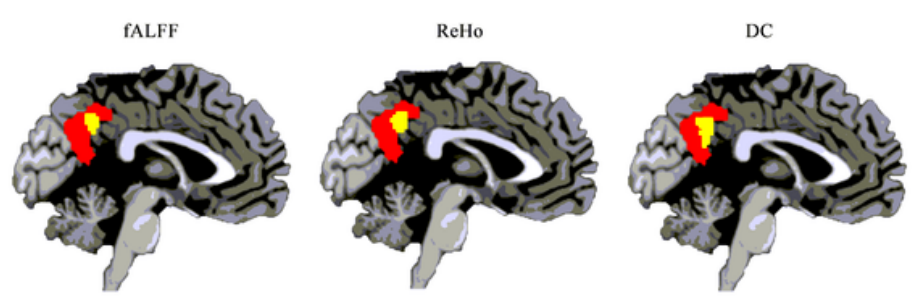

B

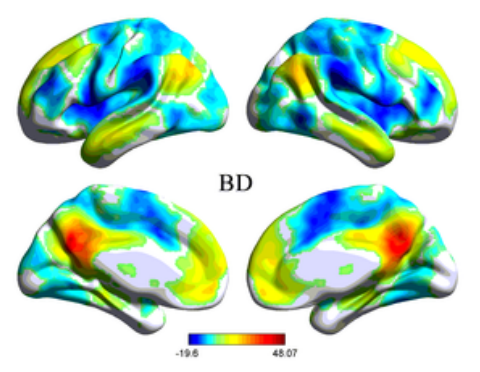

C

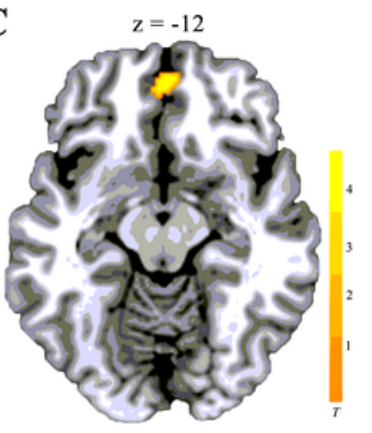

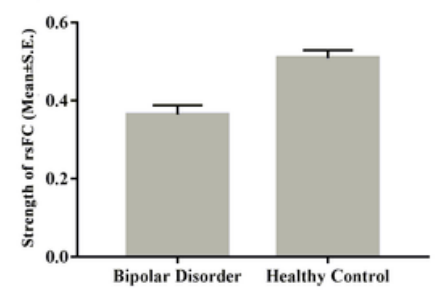

Figure 5

Between-group difference in the rsFC of the left precuneus (No. 153 and 154 in the Brainnetome atlas). Panel A showed the overlapped region between the combined brain regions No. 153 and 154 in the Brainnetome atlas (in red) and the cluster identified by each of functional index (in yellow). Panel B showed the rsFC map within each group. Panel C showed the region whose rsFC with the left precuneus was decreased in patients with BD.

\section{Supplementary Files}

This is a list of supplementary files associated with this preprint. Click to download.

- BIBChecklist.docx 